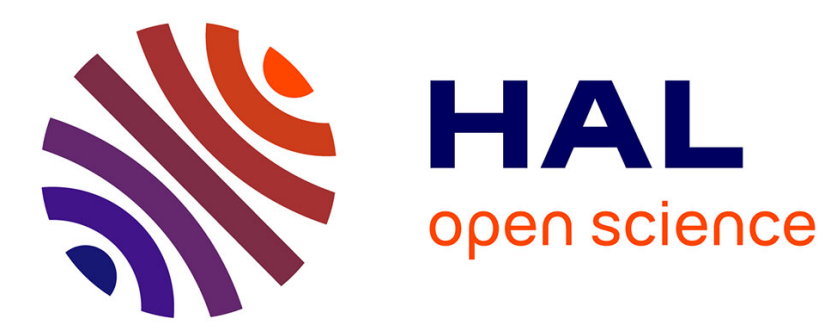

\title{
Nuclear response functions with finite-range Gogny force: Tensor terms and instabilities
}

\author{
A. de Pace, M. Martini
}

\section{To cite this version:}

A. de Pace, M. Martini. Nuclear response functions with finite-range Gogny force: Tensor terms and instabilities. Physical Review C, 2016, 94 (2), pp.024342. 10.1103/PhysRevC.94.024342 . cea01481524

\section{HAL Id: cea-01481524 https://hal-cea.archives-ouvertes.fr/cea-01481524}

Submitted on 2 Mar 2017

HAL is a multi-disciplinary open access archive for the deposit and dissemination of scientific research documents, whether they are published or not. The documents may come from teaching and research institutions in France or abroad, or from public or private research centers.
L'archive ouverte pluridisciplinaire HAL, est destinée au dépôt et à la diffusion de documents scientifiques de niveau recherche, publiés ou non, émanant des établissements d'enseignement et de recherche français ou étrangers, des laboratoires publics ou privés. 


\title{
Nuclear response functions with finite-range Gogny force: Tensor terms and instabilities
}

\author{
A. De Pace \\ Istituto Nazionale di Fisica Nucleare, Sezione di Torino, Via P. Giuria 1, I-10125 Torino, Italy \\ M. Martini \\ ESNT, CEA, IRFU, Service de Physique Nucléaire, Université de Paris-Saclay, F-91191 Gif-sur-Yvette Cedex, France
}

(Received 7 June 2016; published 30 August 2016)

\begin{abstract}
A fully antisymmetrized random phase approximation calculation employing the continued fraction technique is performed to study nuclear matter response functions with the finite-range Gogny force. The most commonly used parameter sets of this force, as well as some recent generalizations that include the tensor terms, are considered and the corresponding response functions are shown. The calculations are performed at first and second order in the continued fraction expansion and the explicit expressions for the second-order tensor contributions are given. Comparisons between first- and second-order continued fraction expansion results are provided. The differences between the responses obtained at the two orders turn out to be more pronounced for the forces including tensor terms than for the standard Gogny ones. In the vector channels the responses calculated with Gogny forces including tensor terms are characterized by a large heterogeneity, reflecting the different choices for the tensor part of the interaction. For the sake of comparison the response functions obtained considering a $G$-matrix-based nuclear interaction are also shown. As a first application of the present calculation, the possible existence of spurious finite-size instabilities of the Gogny forces with or without tensor terms has been investigated. The positive conclusion is that all the Gogny forces but the GT2 one are free of spurious finite-size instabilities. In perspective, the tool developed in the present paper can be inserted in the fitting procedure to construct new Gogny-type forces.
\end{abstract}

DOI: 10.1103/PhysRevC.94.024342

\section{INTRODUCTION}

The past years have been characterized by a regain of interest in nuclear matter response functions in connection with the nuclear structure. The power of this tool to study electron, neutrino, meson, and nucleon scattering on nuclei in the quasielastic peak and beyond (see, for example, Refs. [1-7]) is well known. Recently the formalism of the response functions has been largely employed to investigate the properties of effective nuclear forces. After the seminal work of Ref. [8], a lot of efforts have been made in connection with the Skyrme forces in order to generalize the fully antisymmetrized random phase approximation (RPA) results obtained in that paper, since those calculations were limited to consider the central and the density-dependent terms of the Skyrme interaction. The first generalization was the inclusion in the RPA calculations of the spin-orbit term [9] and after that several papers were devoted to the inclusion of the tensor term [10] and to the investigation of its role [11,12], up to the generalization of the formalism to asymmetric nuclear matter [13]. The effects of other density-dependent terms [14] and of the three-body forces [15] have also been treated. A review on this topic recently appeared [16]. The two main applications of this kind of calculations have been, up to now, the study of the unphysical finite-size instabilities in nuclear energy density functionals [11,12,14,17-19] and the calculation of the neutrino mean free path in nuclear and neutron matter [9,12,14,20-22].

At variance with the case of the Skyrme forces, less attention has been paid to the case of finite-range Gogny forces. The reason is probably twofold. First, in spite of the successes of this kind of force (with which pairing correlations can be automatically taken into account in the mean-field-based calculation, without the introduction of further parameters), the number of mean-field-based calculations using the Gogny force is enormously inferior to the number of corresponding calculations with zero-range Skyrme forces. The second reason is that, due to the finite range of the force, fully analytical calculations of the antisymmetrized RPA nuclear matter response are no longer possible because of the role played by the exchange terms. With finite-range forces analytical results can be achieved only in the so-called ring approximation, which takes into account only the direct contributions, or by considering the Landau-Migdal (LM) limit. The first paper devoted to the RPA response function in infinite nuclear matter employing the Gogny force was the one of Gogny and Padjen [23], which followed the LM approach. An approximation beyond the standard LM one, based on keeping the full momentum dependence in the direct term and making the LM approximation for the exchange term (LAFET), was studied for the Gogny force in Ref. [24] and compared with the results of a method, developed in the same paper, based on an expansion of the BetheSalpeter equation onto a spherical harmonic basis, a method that in principle can be carried out up to any degree of accuracy.

Another approach for the treatment of the fully antisymmetrized RPA response with finite-range nuclear forces is the one based on the continued fraction (CF) technique. Calculations employing this method, for meson-exchangetype potentials, were done in Refs. [25-28] by truncating the CF expansion at first order. The calculation was then pushed up to second order in Ref. [29]. The CF technique up to second order was employed also in Ref. [30], using a Gogny force. Their results support the ones of Ref. [29] on the rapid convergence of the $\mathrm{CF}$ expansion founding, among other 
outcomes, that up to the saturation density the convergence is already achieved at first order.

In the present paper we use the CF technique, following the approach of Ref. [29], and we make use of the Gogny force. Our aim is the study of the nuclear matter response function by employing the most commonly used parametrizations of the Gogny force (D1 [31], D1S [32], D1N [33], and D1M [34]), as well as the recent generalizations that include the tensor terms [35-38]. Several recent papers have shown the crucial role played by the tensor term in the behavior of the nuclear matter response functions [10-13] and, as a consequence, in the finite-size spurious instabilities, but all these studies have only considered Skyrme energy density functionals. Very few works cope with the role of the tensor in the Gogny force. To our knowledge, the only two papers related to this subject are Ref. [39], which performs RPA calculations of the response functions, but in the LM approximation, showing results for the D1MT force, and Ref. [40], which considers, for the D1ST and D1MT interactions, the spin susceptibilities, i.e., the $q \rightarrow 0$, $\omega \rightarrow 0$ limit of the nuclear responses.

\section{FORMALISM}

In the evaluation of response functions, we employ Green's functions techniques, as described, e.g., in Refs. [41,42]. We consider an infinite system of nucleons at a density corresponding to a Fermi momentum $k_{F}$, interacting through a nonrelativistic potential, whose general form, in momentum space, reads

$$
\begin{aligned}
V(\boldsymbol{k})= & V_{0}(k)+V_{\tau}(k) \boldsymbol{\tau}_{1} \cdot \boldsymbol{\tau}_{2}+V_{\sigma}(k) \boldsymbol{\sigma}_{1} \cdot \boldsymbol{\sigma}_{2} \\
& +V_{\sigma \tau}(k) \boldsymbol{\sigma}_{1} \cdot \boldsymbol{\sigma}_{2} \boldsymbol{\tau}_{1} \cdot \boldsymbol{\tau}_{2}+V_{t}(k) S_{12}(\hat{\boldsymbol{k}}) \\
& +V_{t \tau}(k) S_{12}(\hat{\boldsymbol{k}}) \boldsymbol{\tau}_{1} \cdot \boldsymbol{\tau}_{2},
\end{aligned}
$$

where $S_{12}$ is the standard tensor operator and $V_{\alpha}(k)$ represents the momentum space potential in channel $\alpha$ (here we neglect, for the sake of simplicity, the spin-orbit terms).

The response of the system to an external probe can be obtained from the particle-hole (ph) four-point Green's function $G^{\mathrm{ph}}$, which is the outcome of a Galitskii-Migdal integral equation:

$$
\begin{aligned}
G^{\mathrm{ph}}( & K+Q, K ; P+Q, P) \\
= & -G(P+Q) G(P)(2 \pi)^{4} \delta(K-P) \\
& +i G(K+Q) G(K) \int \frac{d^{4} T}{(2 \pi)^{4}} \Gamma^{\mathrm{ph}}(K+Q, K ; T+Q, T) \\
& \times G^{\mathrm{ph}}(T+Q, T ; P+Q, P),
\end{aligned}
$$

$G$ being the exact one-body Green's function and $\Gamma^{\text {ph }}$ the irreducible vertex function in the ph channel (capital letters here refer to four-vectors; lower case letters refer to threevectors). For brevity, in Eq. (2) we have dropped the spinisospin indices.

Out of $G^{\mathrm{ph}}$ one can define the polarization propagator in a given spin-isospin channel $X \equiv(S, M, T)$ :

$$
\begin{aligned}
\Pi_{X}(Q) & \equiv \Pi_{X}(q, \omega) \\
& =i \int \frac{d^{4} P}{(2 \pi)^{4}} \frac{d^{4} K}{(2 \pi)^{4}} G_{X}^{\mathrm{ph}}(K+Q, K ; P+Q, P) .
\end{aligned}
$$

Finally, the system response functions are simply proportional to the imaginary part of $\Pi_{X}$ :

$$
\begin{aligned}
R_{X}(q, \omega) & =-\frac{V}{\pi} \operatorname{Im} \Pi_{X}(q, \omega) \\
& =-\frac{3 \pi A}{2 k_{F}^{3}} \operatorname{Im} \Pi_{X}(q, \omega),
\end{aligned}
$$

$V$ being the volume and $A$ the mass number of the system.

Depending on the approximations done on $G$ and $\Gamma^{\mathrm{ph}}$, one can get different approximations for $\Pi_{X}$. By neglecting $\Gamma^{\mathrm{ph}}$ and dressing the nucleon propagators with the first-order self-energy $\Sigma^{(1)}(k)$ one obtains the Hartree-Fock (HF) or mean-field approximation, $\Pi^{\mathrm{HF}}$. Here we follow the usual approximation of including the mean-field effects through the HF effective mass:

$$
\frac{m^{*}}{m}=\left(1+\left.\frac{m}{k_{F}} \frac{\partial \Sigma^{(1)}(k)}{\partial k}\right|_{k=k_{F}}\right)^{-1},
$$

$m$ being the bare nucleon mass. By replacing in Eq. (2) the irreducible vertex function $\Gamma^{\mathrm{ph}}$ with the matrix elements of the bare potential, one gets the RPA equation for $G^{\mathrm{ph}}$. However, one still has a closed equation only for the full four-point Green's function and not for the simpler polarization propagator.

In order to get the RPA response functions we follow the approach based on the CF expansion of the polarization propagator [29,43-46]. An alternative approach, based on the $\mathrm{CF}$ expansion of the effective interaction, was developed in Refs. [30,47]. Up to second order in the CF expansion (the highest order so far reached in actual calculations) the two approaches are equivalent. Details of the derivation can be found in Ref. [29]. Here we summarize the relevant formulas.

In the CF expansion the RPA polarization propagator reads

$$
\Pi_{\mathrm{X}}^{\mathrm{RPA}}=\frac{\Pi^{\mathrm{HF}}}{1-\Pi_{\mathrm{X}}^{(1) \mathrm{d}} / \Pi^{\mathrm{HF}}-\Pi_{\mathrm{X}}^{(1) \mathrm{ex}} / \Pi^{\mathrm{HF}}-\frac{\Pi_{\mathrm{X}}^{(2) \mathrm{ex}} / \Pi^{\mathrm{HF}}-\left[\Pi_{\mathrm{X}}^{(1) \mathrm{ex}} / \Pi^{\mathrm{HF}}\right]^{2}}{1-\cdots}},
$$

where the expansion has been explicitly shown up to second order. $\Pi_{\mathrm{X}}^{(1) \mathrm{d}}, \Pi_{\mathrm{X}}^{(1) \mathrm{ex}}$, and $\Pi_{\mathrm{X}}^{(2) \mathrm{ex}}$ correspond to the Feynman diagrams in Figs. 1(a), 1(b), and 1(c), respectively.
The $n$th - order exchange propagator in the spin-isospin channel X reads

$$
\Pi_{\mathrm{X}}^{(n) \mathrm{ex}}(q, \omega)=\sum_{\alpha_{i}} C_{\mathrm{X}}^{\alpha_{1}} \ldots C_{\mathrm{X}}^{\alpha_{n}} \Pi_{\alpha_{1} \ldots \alpha_{n}}^{(n) \mathrm{ex}}(q, \omega),
$$




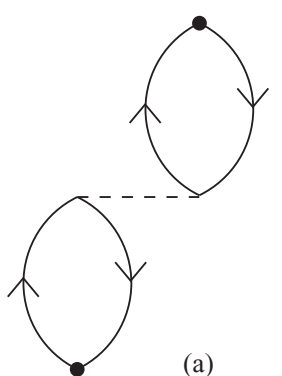

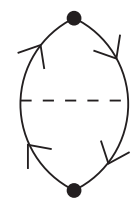

(b)

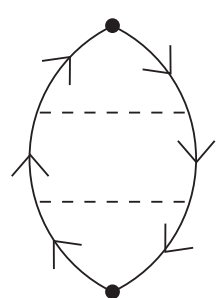

(c)
FIG. 1. Feynman diagrams that enter the second-order CF expansion of the RPA propagator: (a) first-order direct, (b) first-order exchange, and (c) second-order exchange.

where the indices $\alpha_{i}$ run over all the spin-isospin channels of the interaction and all the spin-isospin factors depending on the probed channel are condensed in the coefficients $C_{\mathrm{X}}^{\alpha_{i}}$ (see Table I). Thus, the calculation of the RPA response at order $n$ in the CF expansion is reduced to the calculation of the exchange contributions $\Pi_{\alpha_{1} \ldots \alpha_{n}}^{(n) \text { ex }}$ up to order $n$. Details about the latter in the case of a Gogny interaction are given in the Appendix. Analogous expressions for the case of a meson-exchange potential can be found in the Appendix of Ref. [29].

\section{RESPONSE FUNCTIONS RESULTS}

\section{A. Standard parametrizations of the Gogny force}

The general expression of the Gogny interaction in the coordinate space is

$$
\begin{aligned}
V(\boldsymbol{r})= & \sum_{j=1}^{2}\left(W_{j}+B_{j} P_{\sigma}-H_{j} P_{\tau}-M_{j} P_{\sigma} P_{\tau}\right) e^{-\frac{r^{2}}{\mu_{j}^{2}}} \\
& +t_{0}\left(1+x_{0} P_{\sigma}\right) \rho^{\alpha_{0}} \delta(\boldsymbol{r}),
\end{aligned}
$$

where $\boldsymbol{r}$ is the distance between two nucleons. The first term of Eq. (8) is given by a sum of two Gaussians with effective range $\mu_{1}$ and $\mu_{2}$ simulating the short- and long-range components of a realistic interaction in the nuclear medium. This finite-range term includes all possible mixtures of spin and isospin operators, being $P_{\sigma}=\left(1+\sigma_{1} \cdot \sigma_{2}\right) / 2$ and $P_{\tau}=\left(1+\tau_{1} \cdot \tau_{2}\right) / 2$ the spin and isospin exchange operators, respectively. The second term is the zero-range density-dependent contribution. Usually the Gogny force also contains a zero-range spin-orbit term. We omit it, as is generally done in the nuclear matter calculations

TABLE I. The spin-isospin coefficients $C_{X}^{\alpha}$ (see text), in the various spin-isospin channels, for the interaction of Eq. (1).

\begin{tabular}{llrrrrr}
\hline \hline$X \equiv(S, M, T)$ & $C_{X}^{0}$ & $C_{X}^{\tau}$ & $C_{X}^{\sigma}$ & $C_{X}^{\sigma \tau}$ & $C_{X}^{t}$ & $C_{X}^{t \tau}$ \\
\hline$(0,0,0)$ & 1 & 3 & 3 & 9 & 0 & 0 \\
$(0,0,1)$ & 1 & -1 & 3 & -3 & 0 & 0 \\
$(1,1,0)$ & 1 & 3 & -1 & -3 & -1 & -3 \\
$(1,1,1)$ & 1 & -1 & -1 & 1 & -1 & 1 \\
$(1,0,0)$ & 1 & 3 & -1 & -3 & 2 & 6 \\
$(1,0,1)$ & 1 & -1 & -1 & 1 & 2 & -2 \\
\hline \hline
\end{tabular}

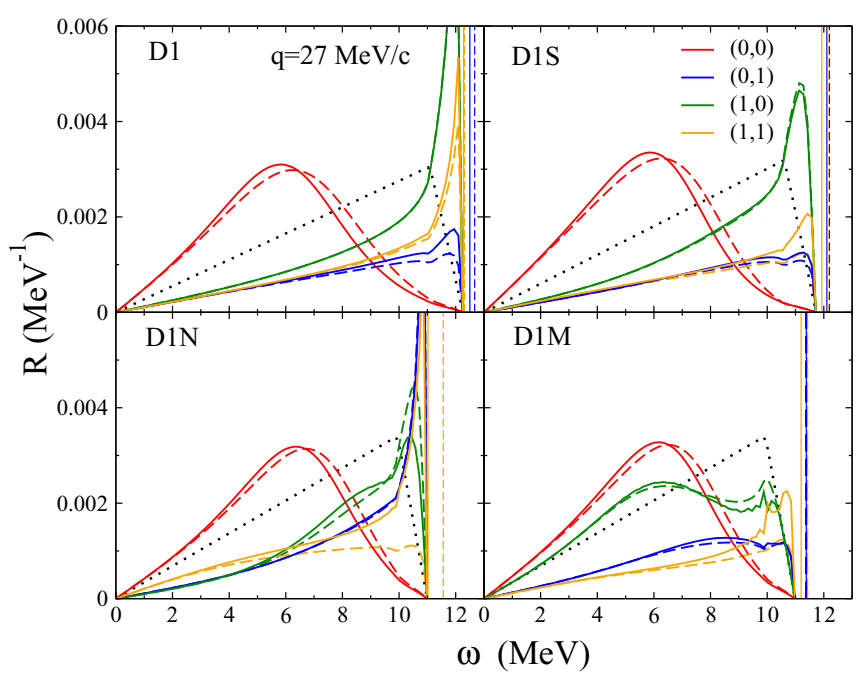

FIG. 2. RPA response function in symmetric nuclear matter at $k_{F}=270 \mathrm{MeV} / c$ and $q=27 \mathrm{MeV} / c$ calculated with the continued fraction technique at first order (dashed line) and at second order (solid line) in the CF expansion by considering different parametrizations of the Gogny interaction. The different spin and isospin channels $(S, T)$ are plotted in different colors. The black dotted lines represent the HF response. The collective modes above the continuum are represented by vertical lines.

[24,30,48-50]. Indeed, in Ref. [9] it was shown that the effects of the spin-orbit interaction on the calculations of the nuclear response functions are small, even at momentum transfer larger than the Fermi momentum. This conclusion obtained by using Skyrme-type forces is expected to remain valid also for the Gogny forces, due to the similarity of the spin-orbit term in the two cases. The expression of the Gogny interaction given in Eq. (8) is the one corresponding to the most commonly used parametrizations, such as D1, D1S, D1N, and D1M. For the values of the parameters $\mu_{j}, W_{j}, B_{j}, H_{j}, M_{j}, t_{0}, x_{0}$, and $\alpha_{0}$ corresponding to these four forces see, for example, Ref. [51].

Let us consider now the RPA response functions $R_{X}(q, \omega)$ in the four spin and isospin channels calculated at first and second order in the $\mathrm{CF}$ expansion using the four parametrizations of the Gogny interaction mentioned above. For the sake of illustration we show these responses in Figs. 2 and 3 for the fixed value of $k_{F}=270 \mathrm{MeV} / c$ and for two values of the momentum transfer, $q=27$ and $q=270 \mathrm{MeV} / c$. We consider these values in order to compare our results with the ones obtained in Ref. [30] where only the D1 parametrization was considered. Starting with this interaction one can observe that our results are similar to the ones obtained in Ref. [30] (we remind that there is a global multiplicative factor $4 \rho_{0} \hbar c$ between our results and the ones of Ref. [30]). In analogy with Ref. [30] we can conclude that for the D1 parametrization the first order in the $\mathrm{CF}$ expansion gives a reliable description of the response functions for the $(S, T)$ spin and isospin channels $(1,0)$ and $(1,1)$, but not for the $(0,0)$ channel, where it is necessary to include the second order. For the $(0,1)$ channel, while at $q=270 \mathrm{MeV} / c$ the results of our work are once again similar to the ones of Ref. [30] (and are characterized by a small effect between the two orders of the 


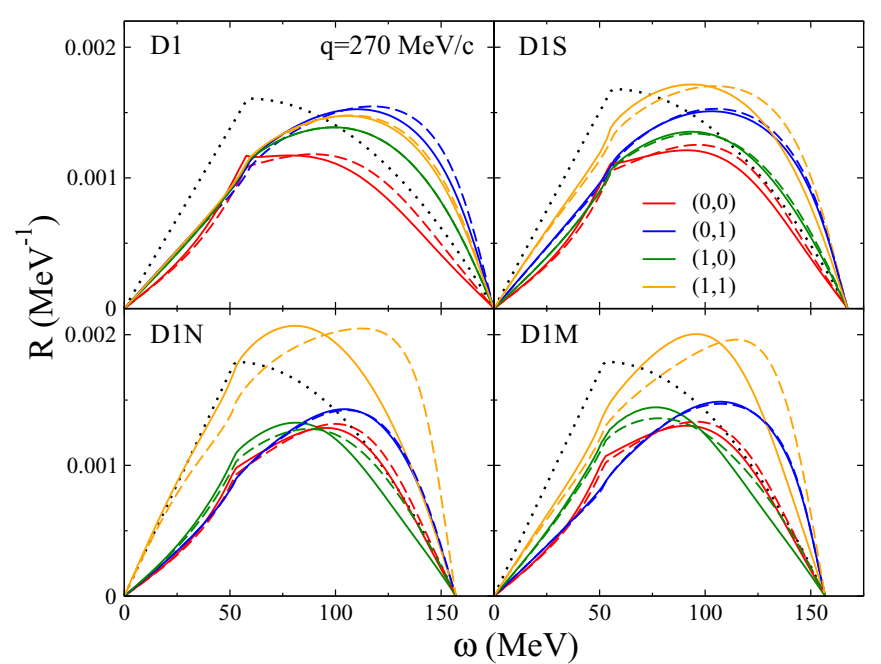

FIG. 3. As in Fig. 2, but for $q=270 \mathrm{MeV} / c$.

CF expansion), some differences between the two calculations appear at $q=27 \mathrm{MeV} / c$. In our case, the collective mode above the particle-hole continuum region remains outside the continuum even at second order in the CF expansion. It does not seem to be the case in the calculations of Ref. [30].

Turning to the other three interactions, whose results for the different response functions are presented here for the first time, we can make two general comments:

(1) In the $(0,0)$ channel the responses calculated with the different parametrizations show a similar qualitative behavior, not only when compared to each other but also with respect to the $\mathrm{HF}$ results; moreover, the difference between the first- and second-order $\mathrm{CF}$ expansion results is similar for the four forces.

(2) In the other $(S, T)$ channels, the various parametrizations can show important differences in the transferredenergy dependence; the convergence of the $\mathrm{CF}$ expansion as well strongly depends on the force parameters in the different $(S, T)$ channels.

Considering now specific parametrizations, we can affirm, qualitatively speaking, that the results obtained with D1S are not so different from the ones obtained with D1. For these two parametrizations, some difference can be observed in the $(1,1)$ channel. Indeed, at $q=27 \mathrm{MeV} / c$ the collective mode is above the ph continuum for D1S, but not for D1. At $q=270 \mathrm{MeV} / c$ the response is quenched with respect to the HF one in the case of D1, while there is a small enhancement for the D1S case. In this $(1,1)$ channel, the difference between first and second order in the CF expansion is more pronounced for D1S than for D1.

Remaining on this $(1,1)$ channel, the enhancement of the responses at $q=270 \mathrm{MeV} / c$ with respect to the $\mathrm{HF}$ case, as well as the discrepancy between first and second order in the CF expansion, are largely evident for D1M and D1N.

Without entering into the details of the behavior of the responses in the different channels for each parametrization, we just mention that at $q=27 \mathrm{MeV} / c$ the response in the $(1,0)$ channel calculated with D1M is quite different with respect to the other parameterizations.

In the $(0,1)$ channel the results strongly depend on the appearance (like in the case of D1, D1S, and D1M) or not (like in the case of D1N) of the collective mode above the ph continuum. This collective mode in the $S=0, T=1$ channel is the counterpart of the giant dipole resonance (GDR) in finite nuclei. A large-scale theoretical quasiparticle RPA calculation of dipole excitations in the whole nuclear chart was performed in Ref. [52] where the D1S and D1M Gogny forces are used. A systematic shift toward lower energies is found for the GDR mode calculated with D1M with respect to D1S. An equivalent shift appears in the present infinite nuclear matter results. Lower energy collective modes for D1M when compared to D1S are also found in the $(1,1)$ channel, here for infinite nuclear matter, and in Ref. [53] for the Gamow-Teller resonances of finite nuclei.

Beyond the discussed differences related to the use of different parameter sets of the Gogny force, an important and general comment is in order: independently of $q$, of the choice for the parametrization of the interaction, and of the spin-isospin channel of excitation, one can observe that at low $\omega$ the difference between the results obtained at first order in the CF expansion and the ones obtained at second order is always small. This is particularly important for studies of the finite-size instabilities of the nuclear matter, that are discussed in Sec. IV, which involve calculations of the response functions at $\omega=0$.

\section{B. Gogny force with tensor terms}

Some Gogny-type forces, less used up to now in the literature, are characterized by the presence of additional tensor terms. This is the case of the GT2 force [35] in which a tensorisovector contribution of Gaussian form is added to the central channels of Eq. (8). Tensor terms of Gaussian form appear also in D1ST2a and D1ST2b [37] as well as in D1ST2c and D1MT2c [38], where beyond a tensor-isovector component a tensor-isoscalar one is included. Another important difference between the GT2 force and these last four parametrizations is that in the GT2 case the inclusion of a tensor term involved a refitting of all the parameters, whereas for the other cases the tensor terms have been added to the D1S or D1M without changing the values of the central parameters. In the same spirit also the D1ST and D1MT interactions were introduced [36]. In this case the radial part of the additional tensor-isospin term was based on the analogous one in the microscopic Argonne V18 interaction; hence, it was not characterized by a Gaussian behavior. To include also these D1ST and D1MT interactions in our analysis, which is based on Gaussian interactions, we have fitted the tensor component of the D1MT and D1ST interactions with a sum of three Gaussians.

Turning to the nuclear responses, we show in Figs. 4 and 5 the results obtained at first and second order in the CF expansion by employing the D1ST, D1ST2(a,b,c), D1MT, D1MT2c, and GT2 Gogny interactions. For the sake of comparison we also display the response functions calculated at first order employing a $G$-matrix-based nuclear interaction. This interaction is based on the $G$-matrix calculation of Ref. [54] and it has been employed in RPA calculations of 


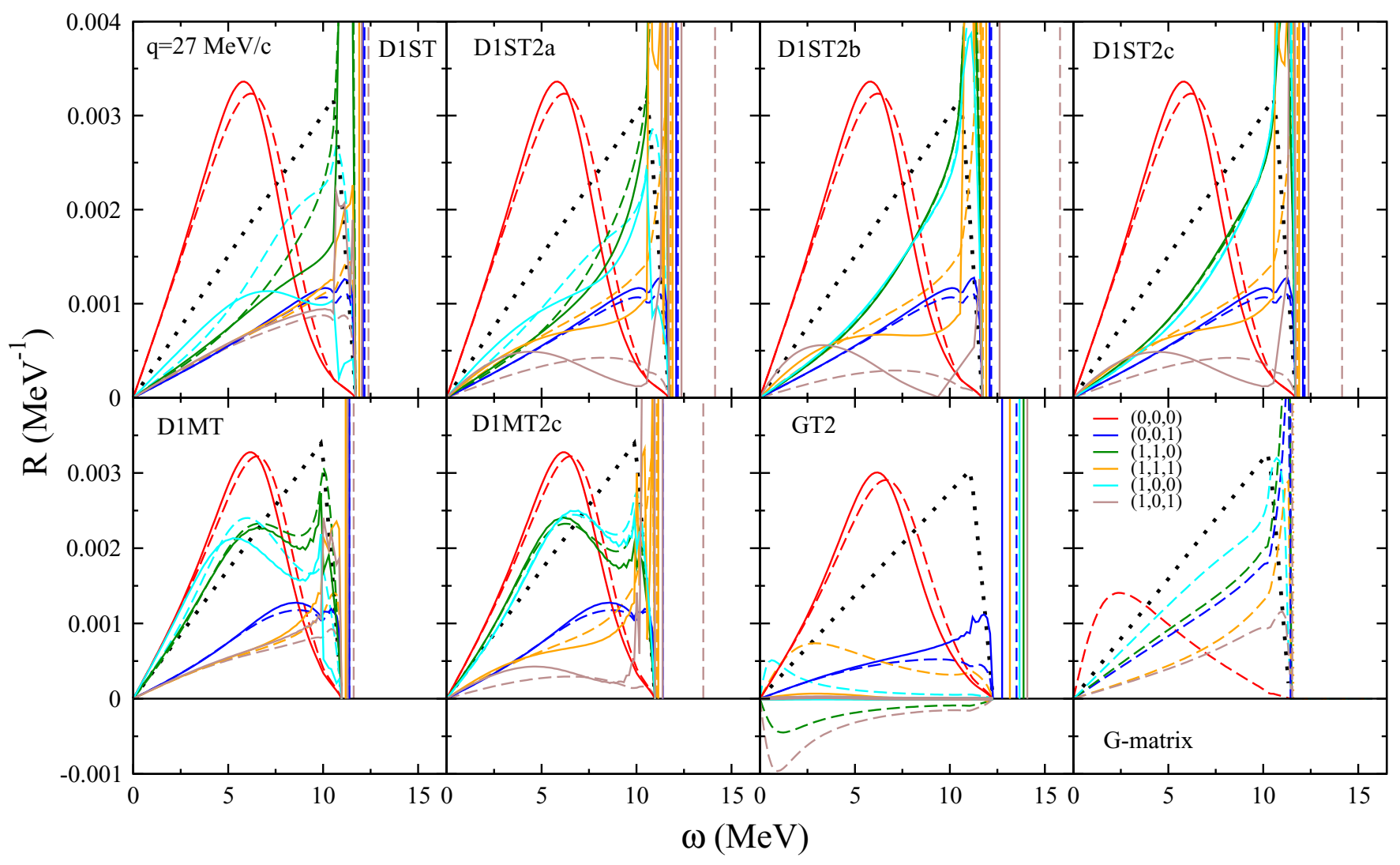

FIG. 4. RPA response function in symmetric nuclear matter at $k_{F}=270 \mathrm{MeV} / c$ and $q=27 \mathrm{MeV} / c$ calculated with the continued fraction technique at first order (dashed line) and at second order (solid line) in the CF expansion by considering different parametrizations of the Gogny interaction with tensor terms. The different spin and isospin channels $(S, M, T)$ are plotted in different colors. The black dotted lines represent the HF response. The collective modes above the continuum are represented by vertical lines. For comparison, we also display the response functions calculated with a $G$-matrix nuclear interaction.

quasielastic response functions both in finite nuclei $[5,55,56]$ and in nuclear matter [29]. For $k_{F}$ and $q$ we choose the same values $\left(k_{F}=270 \mathrm{MeV} / c ; q=27\right.$ and $\left.q=270 \mathrm{MeV} / c\right)$ as the ones considered for Figs. 2 and 3.

The responses shown in Figs. 4 and 5 have never been calculated before by considering the CF expansion approximation. However, in the case of the D1MT interaction we can compare our results with the ones published in Ref. [39], where the responses for the D1MT interaction were calculated in the Landau framework by truncating the residual interaction at $l_{\max }=3$. Our results for $q \simeq 0.1 k_{F}$ are plotted in the left lower panel of Fig. 4 and should be compared with the results of Fig. 3 of Ref. [39]. The agreement is fairly good in all the $(S, M, T)$ channels. The agreement between the two approaches no longer holds at $q \simeq k_{F}$, as one can notice by comparing our results in the left lower panel of Fig. 5 with the results of Fig. 4 of Ref. [39]. The only channels where the two calculations seem to be in agreement are the $(S, M, T)=(0,0,1)$ and, perhaps, $(1,1,1)$ ones. In all the other channels there are differences, more or less pronounced, in the shape of the responses, in the position of the peak, and in the behavior with respect to the HF results. The good agreement between the calculations at $q \simeq 0.1 k_{F}$, but not at $q \simeq k_{F}$, seems to suggest that truncating the expansion of the residual interaction at $l_{\max }=3$ is not enough at large $q$. This conclusion is independent of the presence of the tensor terms, since the disagreement appears also in the $S=0$ channel and since the discrepancy survives also in the comparison with the D1M parametrization (which does not contain tensor terms), as seen from the results presented in the bottom right panel of our Fig. 3 and in Fig. 5 of Ref. [39]. One should mention that there are small differences in the choice of the effective mass and of the momentum transfer between our figures and the ones of Ref. [39], as one can notice for example by the different end point $\left(\omega=\frac{q^{2}}{2 m^{\star}}+\frac{q k_{F}}{m^{\star}}\right)$ of the response functions. We have repeated the calculations by choosing the same values of $q$ and $m^{\star}$ as Ref. [39]. The behavior of our curves and, hence, our conclusions remain the same.

Turning to a global discussion of the responses obtained with the different Gogny forces containing tensor terms, the following comments are in order:

(i) One observes in general homogeneity of results in the $S=0$ channels and some heterogeneity in the $S=1$ ones.

(ii) The $(S, M, T)=(0,0,0)$ responses obtained with the different Gogny forces are in qualitative agreement among them, for $q=27 \mathrm{MeV} / c$ as well as for $q=270 \mathrm{MeV} / c$, but differ from the ones obtained with the $G$-matrix interaction which were shown [5] 


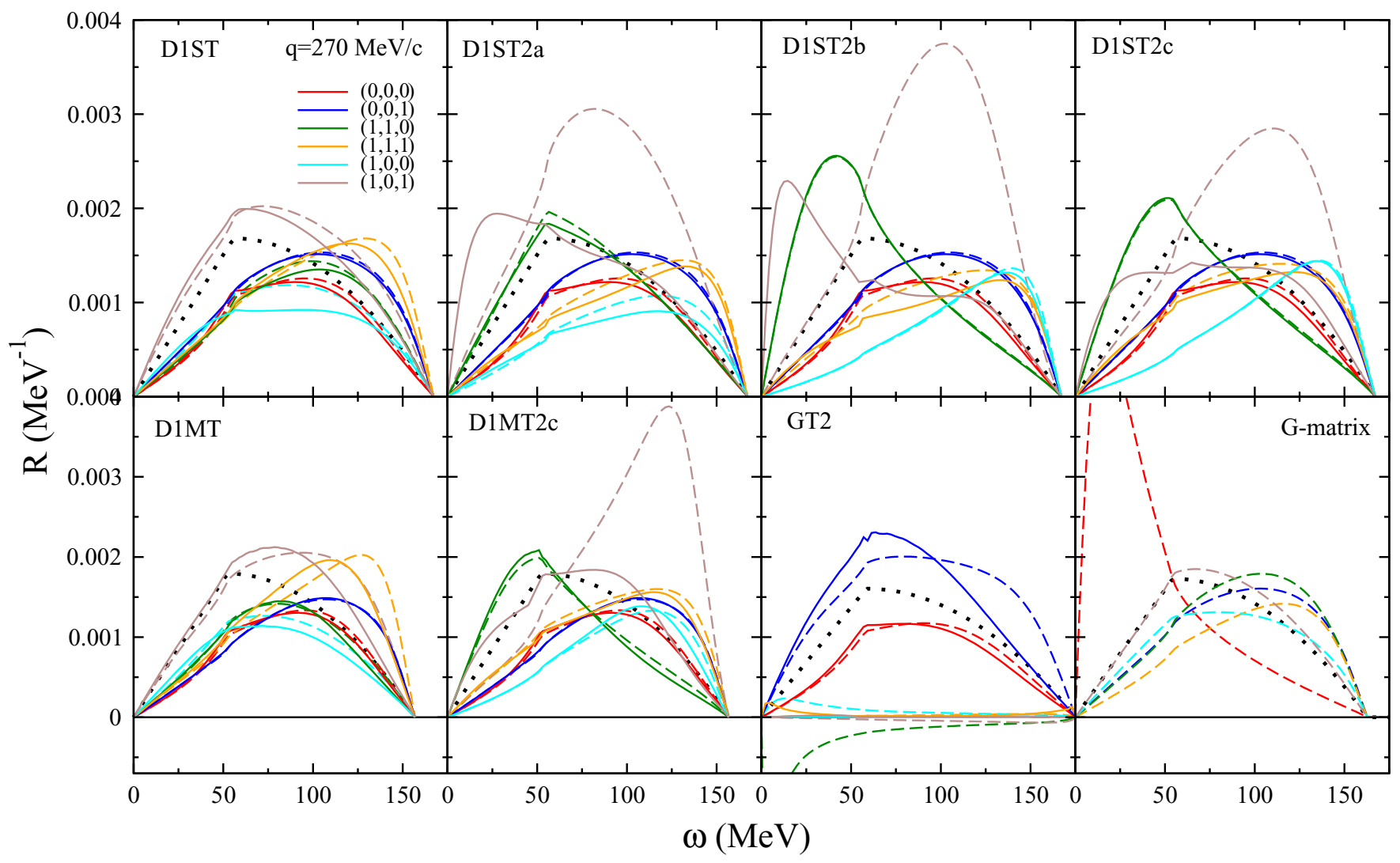

FIG. 5. As in Fig. 4, but for $q=270 \mathrm{MeV} / c$.

to successfully reproduce the $\mathrm{K}^{+}$-nucleus quasielastic cross section, largely dominated by the scalarisoscalar channel, at $q=290,390$, and $480 \mathrm{MeV} / c$.

(iii) The $(S, M, T)=(0,0,1)$ responses as well present always similar features: they are characterized by a strong quenching of the continuum and the appearance of a collective mode at $q=27 \mathrm{MeV} / \mathrm{c}$ and by some quenching and hardening (except for the GT2 case) at $q=270 \mathrm{MeV} / c$. Similar features characterize also the $G$-matrix results, even if in this case at $q=27 \mathrm{MeV} / c$ the collective mode enters in the continuum.

(iv) Concerning the $S=1$ channels, a first remark is about the unphysical result of negative responses in some cases with the GT2 force, when calculated at first order in $\mathrm{CF}$, which reflects a lack of convergence of the CF method when one considers this interaction. Furthermore, always for GT2, at second order in the CF expansion all the $S=1$ responses are totally quenched and characterized by a collective mode at $q=27 \mathrm{MeV} / c$. This total quenching remains also at $q=270 \mathrm{MeV} / c$.

(v) For the other interactions one can observe that at $q=27 \mathrm{MeV} / c$ the differences in the $(S, M, T)=$ $(1,1,0)$ and $(S, M, T)=(1,0,0)$ channels, for responses calculated with the D1ST* and D1MT* forces, essentially reflect the differences already present in the $(S, T)=(1,0)$ channel between D1S and D1M. At $q=270 \mathrm{MeV} / c$ the split between the
$(S, M, T)=(1,1,0)$ and $(S, M, T)=(1,0,0)$ results is more or less pronounced depending on the interaction. The two forces giving the results closer to the $G$-matrix calculations are, for these channels, D1ST and D1MT.

(vi) The responses in the $(S, M, T)=(1,0,1)$ channel always present at $q=27 \mathrm{MeV} / c$ a collective mode above the continuum, also in the $G$-matrix case, while they can be very different from each other at $q=270 \mathrm{MeV} / c$.

(vii) In the $(S, M, T)=(1,1,1)$ channel at $q=27 \mathrm{MeV} / c$ the collective mode can be above or inside the continuum, depending on the interaction; at $q=$ $270 \mathrm{MeV} / c$, however, the qualitative behavior of all the responses is always very similar (except, as usual, for the GT2 case) and in agreement with the $G$-matrix results. This agreement opens the perspective of using these Gogny-type forces in the calculation of the neutrino-nucleus cross sections (and of the neutrino mean-free path in nuclear matter), which are dominated by the spin-isospin transverse response $[7,12,57]$.

(viii) The differences between the results obtained at first and second order in the CF expansion are in general more pronounced for these forces including tensor terms with respect to the case of the standard parametrizations of the Gogny forces. These differences are often very pronounced, in particular in the $(S, M, T)=(1,0,1)$ case, for $q=27 \mathrm{MeV} / c$ 
as well as for $q=270 \mathrm{MeV} / c$ and remain also in the $\omega \rightarrow 0$ limit, important for the calculations of the instabilities.

\section{FINITE-SIZE INSTABILITIES}

The study of the unphysical finite-size instabilities through the nuclear matter response function formalism has attracted a lot of interest in the past years starting from the work of Ref. [17]. Many recent investigations have been performed by considering Skyrme-type nuclear energy density functionals [11,12,14,17-19]. No studies of finite-size instabilities with the Gogny forces have been published up to now.

In the case of Skyrme functionals, in Ref. [17] a qualitative link was suggested between the appearance of finite-size instabilities of nuclear matter near saturation density and the impossibility to converge for self-consistent calculations in finite nuclei using some parametrizations of the nuclear energy density functionals. More precisely it has been shown that, using the SkP and LNS parameter sets, the neutron and proton densities were characterized by strong and opposing oscillating behavior, which increased with the number of iterations of the self-consistent procedure. As the one-body equations of motion are solved iteratively, an instability in the scalar isovector channel occurs when it becomes energetically favorable to build oscillations of neutrons against protons of unlimited amplitude. In Ref. [17] it was also shown that the same SkP and LNS parametrizations lead to divergences of the nuclear matter response functions at $\omega=0$ and finite $q$ when calculated in the $S=0, T=1$ channel for densities close to the saturation one. These are the critical densities $\rho_{c}$, i.e., the lowest densities at which the nuclear response calculated at zero transferred energy exhibits a pole.

After the suggestion of the qualitative link between the finite nuclei and the nuclear matter phenomena described above, several papers have been devoted to the calculations of the critical densities at finite $q$ in nuclear and neutron matter of many zero-range nuclear energy density functionals, including or not the tensor components [11,12,14].

A systematic quantitative analysis of the connection between the finite nuclei and nuclear matter instabilities in the $S=0, T=1$ channel was performed in Ref. [18], finding that a functional is stable if the lowest critical density at which a pole occurs in nuclear matter calculations is larger than the central density of ${ }^{40} \mathrm{Ca}$, in practice around 1.2 times the saturation density. In addition, one has also to verify that this pole represents a distinct global minimum in the $\left(\rho_{c}, q\right)$ plane. This criterion can be incorporated into the fitting procedure of the coupling constants of the energy density functionals and has the advantage of being based on computationally friendly nuclear matter calculations.

In Ref. [19] the quantitative analysis was extended to the $S=1$ channel by studying not only ground-state properties, but also vibrational excited states of finite nuclei. The stability criterion mentioned above, derived in Ref. [18] for the $S=0$, $T=1$ channel, was found to remain valid also in the $S=$ 1 channel. We remind that the $S=0, T=0$ channel is characterized by the physical spinodal instability; hence, it is not considered in the studies of unphysical instabilities.

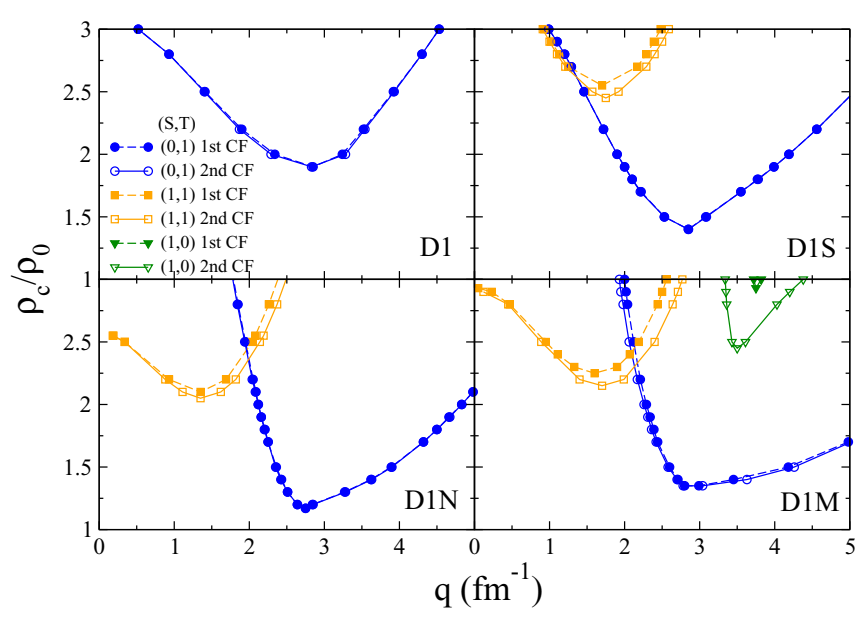

FIG. 6. Critical densities $\rho_{c}$ divided by a constant value of the saturation density $\rho_{0}=0.16 \mathrm{fm}^{-3}$ as a function of the transferred momentum $q$ (in $\mathrm{fm}^{-1}$ ) for the most commonly used parametrizations of the Gogny force. The calculations of $R_{(S, T)}(q, \omega=0)$, through which the critical densities are deduced, are performed at first and second order in the continued fraction expansion.

All the studies described above considered Skyrme functionals. Turning to the Gogny interaction, the stability studies involving nuclear matter calculations have considered up to now only the $q \rightarrow 0$ limit, corresponding to perturbation of infinite wavelength, and have used the well-known stability conditions established by Migdal [58], starting from the seminal work of Gogny and Padjen [23]. In this context, beyond the $(S, T)=(0,0)$ channel, where the stability condition is not satisfied at low densities, reflecting the existence of the well-known spinodal instability, in the other channels the infinite wavelength $(q=0)$ instabilities of the most commonly used Gogny interactions typically appears for densities larger than two or three times the nuclear matter saturation density $\rho_{0}$. For example, in the D1N case the lowest density instability appears in the $(1,1)$ channel at $\rho_{C} \simeq 2.5 \rho_{0}$, while for D1S it appears in the $(0,1)$ channel at $\rho_{C} \simeq 3.5 \rho_{0}$.

Here we consider for the first time the evolution of the critical densities with the momentum transfer $q$. Discarding the $(0,0)$ channel and its corresponding spinodal instability, we start by showing in Fig. 6 the critical densities in the other $(S, T)$ channels as a function of $q$ for the four most commonly used parametrizations of the Gogny force.

In the D1 case only the $R_{(0,1)}(q, \omega=0)$ exhibits a pole at finite $q$. The corresponding critical density is never lower than $2 \rho_{0}$. For the other three Gogny parametrizations (D1S, D1N, and D1M) the poles appear at finite $q$ not only in the $(0,1)$ channel, but also in the $(1,1)$ one [and in the $(1,0)$ for D1M]. This $(1,1)$ channel, even if it presents critical densities lower than $3 \rho_{0}$ already for $q=0$ in the case of D1N and D1M, is characterized by a relatively smooth decrease of $\rho_{C}$ with $q$ and in any case for the D1S, D1N, and D1M the corresponding critical densities are never lower than $2 \rho_{0}$, even at large $q$. On the contrary, for the $(0,1)$ channel the critical densities rapidly decrease with $q$ reaching values around $\rho_{C} \simeq 1.5 \rho_{0}$ for D1S and $\mathrm{D} 1 \mathrm{M}$ and around $\rho_{C} \simeq 1.2 \rho_{0}$ for D1N. All the curves 


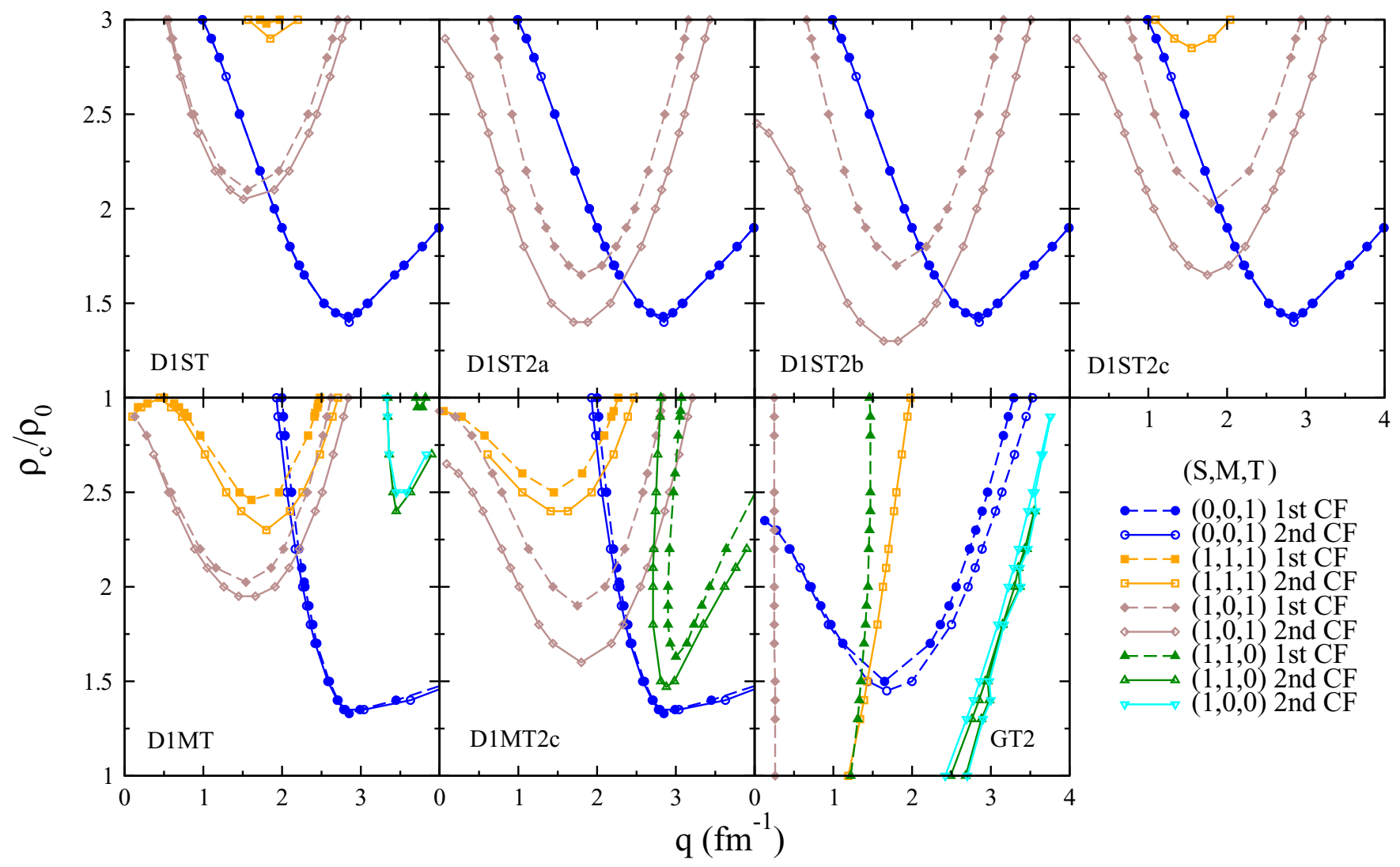

FIG. 7. As in Fig. 6, but for the Gogny forces with tensor terms.

obtained are furthermore characterized by a global minimum in the $\left(\rho_{c}, q\right)$ plane. The two stability criteria established in Ref. [18] are thus satisfied by the most commonly used Gogny forces. Hence they are free from instability problems. The only case to be treated with some caution is the one of the D1N parametrization, which in any case is rarely used in the finite nuclei calculations. The case of $\mathrm{D} 1 \mathrm{~N}$, together with $\mathrm{D} 1 \mathrm{M}$, presents also some small differences in the critical densities between the results obtained by calculating the nuclear response functions at first order in the CF expansion and the results at second order, while for the D1 and D1S cases the results at the two orders practically coincide. For all these four interactions we can anyway conclude that the calculation at first order in the CF expansion can be considered enough for finite-size instabilities studies.

Turning to the parametrizations of the Gogny forces including tensor terms, we show in Fig. 7 the results for the critical densities as a function of the momentum transfer calculated at first and second order in the CF expansions for the D1ST, D1ST2(a,b,c) D1MT, D1MT2c, and GT2 interactions.

In the case of D1MT, in the $q \rightarrow 0$ limit we find for the critical density in the $(1,1)$ channel a value close to $\rho_{c}=0.45 \mathrm{fm}^{-3}$, a result already obtained in Ref. [40] in the context of spin susceptibilities calculations. This is the only point with which we can compare our calculations including tensor terms.

As in the case without tensor terms, finite-size instabilities appear only in the $T=1$ channels for the D1ST*-type force, while they appear in the $T=1$ channels and also for
$(S, M, T)=(1,1,0)$ for the D1MT*-type forces. The behavior of the critical densities in the $(S, M, T)=(0,0,1)$ channel for the D1ST*- and D1MT*-type forces reflects the one of D1S and D1M. In the $S=1, T=1$ channel the presence of tensor terms lowers (raises) the values of the critical densities for the $M=0(M=1)$ component, when compared to the corresponding cases without tensor.

In the $S=1$ channels differences between the results obtained at first and second order in the CF expansion appear. In any case the two stability criteria established in Ref. [18] are always satisfied by all the parametrizations of the D1ST*- and D1MT*-type Gogny forces considered here in all the $(S, M, T)$ channels.

This reassuring result no longer holds for the GT2 force, which presents finite $q$ instabilities at any density in the $(S, M, T)=(1,1,0)$ and $(S, M, T)=(1,0,1)$ channels. These results are the counterpart of the peculiar behavior of the response functions calculated with this GT2 force: negative at first order in the CF expansion and with a ph continuum totally quenched at second order in the CF expansion.

\section{CONCLUSION}

We have studied RPA nuclear matter response functions by considering the nucleons interacting via the finite-range Gogny force. We have considered the most commonly used parametrizations of this force, as well as some recent generalizations that include the tensor terms. We have performed a fully antisymmetrized RPA calculation, including, i.e., the 
exchange contribution of the particle-hole interaction, by employing the $\mathrm{CF}$ technique. The calculations were performed by truncating the continued fraction expansion at first and second order, the highest one so far reached in the context of finite-range forces.

Concerning the most commonly used parameter sets of the Gogny force, we obtained results similar to the ones presented in Ref. [30] for the D1 interaction, the only interaction considered in that paper. The response functions in the four $(S, T)$ channels obtained with the other common parameter sets, namely D1S, D1N, and D1M, are presented here for the first time. Some differences in the transferred-energy behavior appear, in particular at small momentum transfer, depending on the chosen parametrization. The convergence of the $\mathrm{CF}$ expansion as well strongly depends on the force parameters.

Concerning the Gogny forces including tensor terms, we considered the D1ST, D1ST2(a,b,c), D1MT, D1MT2c, and GT2 interactions. In the case of the D1MT interaction we compared our results with the ones published in Ref. [39], where the responses for the D1MT interaction were calculated in the Landau framework by truncating the residual interaction up to $l_{\max }=3$. For all the other interactions including the tensor terms, the response functions appear here for the first time. A general homogeneity characterizes the $S=0$ channels, while in the $S=1$ channel a heterogeneity of the results appears, reflecting the very different choices for the tensor terms. Furthermore, the differences between the results obtained at first and second order in the CF expansion turn out to be more pronounced for the forces including tensor terms with respect to the case of the standard Gogny ones.

An interesting point is the behavior of the responses calculated with the difference forces including the tensor terms in the $(S, M, T)=(1,1,1)$ channel, which is similar for all the parametrizations and in agreement with the $G$-matrix results. This agreement opens the perspective of using these Gognytype forces in the calculation of the neutrino-nucleus cross sections (and of the neutrino mean free path in nuclear matter), which are dominated by the spin-isospin transverse response. The correspondence between continuum RPA calculations in finite nuclei and nuclear matter results in the quasielastic electron and neutrino scattering is illustrated, for example, in Refs. [59,60].

Continuum RPA calculations in finite nuclei using the Gogny force were developed in Ref. [61] and recently generalized to the charge-exchange excitations in Ref. [62]. It would be very interesting to compare the finding of that approach with the one developed in the present paper (employing a same Gogny interaction with or without tensor terms) in order to investigate up to where one can push a nuclear matter approach to study finite nuclei properties and reactions.

As a first instance of connection between nuclear matter and finite nuclei, we have employed the present nuclear matter approach to study the spurious finite-size instabilities. We have shown for the first time that, at variance with some zero-range
Skyrme functionals, the most commonly used finite-range D1, D1S, and D1M Gogny forces satisfy the stability criteria of Ref. [18] in all the $(S, T)$ channels; hence, they are free of spurious finite-size instabilities. The only case to be treated with some caution is the one of the D1N parametrization, which in any case is rarely used in the finite nuclei calculations.

The stability criteria of Ref. [18] are also satisfied in all the $(S, M, T)$ channels by all the Gogny forces including tensor terms of type D1MT* and D1ST*. On the contrary, at least at first and second order in the CF expansion, the GT2 force is unstable in all the $S=1$ channels. However, this result might just signal the poor convergence of the $\mathrm{CF}$ expansion for the GT2 interaction, given the extremely strong tensor component which is present in this force.

In perspective, the tool developed in the present paper could be inserted in the fitting procedure to construct new Gognytype forces. It would be also interesting to repeat the study of the present paper by considering the recently developed D2 Gogny force [63], characterized by a finite-range densitydependent term, as well as other finite-range forces [64-66] employed for low-energy nuclear structure calculations.

\section{ACKNOWLEDGMENTS}

M.M. acknowledges the support and the framework of the "Espace de Structure et de réactions Nucléaire Théorique" (ESNT, http://esnt.cea.fr) at CEA as well as the hospitality of the INFN-Torino where part of this work was done.

\section{APPENDIX: CONTINUED FRACTIONS WITH THE GOGNY INTERACTION}

We give here the explicit expressions for the first- and second-order exchange diagrams, based on the potential of Eq. (1), where for $V_{\alpha}(k)$ we take the Gaussian expression typical of the Gogny forces, the label $\alpha$ standing for the spin-isospin channel.

After some manipulations, as explained in detail in Ref. [29], the first-order polarization propagator can be cast into the following form:

$$
\begin{aligned}
\Pi_{\alpha}^{(1) \mathrm{ex}}(q, \omega)= & -\left(\frac{m}{q}\right)^{2} \frac{k_{F}^{4}}{(2 \pi)^{4}}\left[\mathcal{Q}_{\alpha}^{(1)}(0, \psi)-\mathcal{Q}_{\alpha}^{(1)}(\bar{q}, \psi)\right. \\
& \left.+\mathcal{Q}_{\alpha}^{(1)}(0, \psi+\bar{q})-\mathcal{Q}_{\alpha}^{(1)}(-\bar{q}, \psi+\bar{q})\right],
\end{aligned}
$$

where

$$
\begin{aligned}
\mathcal{Q}_{\alpha}^{(1)}(\bar{q}, \psi)= & 2 \int_{-1}^{1} d y \frac{1}{\psi-y+i \eta_{\omega}} \\
& \times \int_{-1}^{1} d y^{\prime} W_{\alpha}^{\prime \prime}\left(y, y^{\prime} ; \bar{q}\right) \frac{1}{y-y^{\prime}+\bar{q}},
\end{aligned}
$$

whereas for the second-order polarization propagator one has

$$
\begin{aligned}
\Pi_{\alpha \alpha^{\prime}}^{(2) \mathrm{ex}}(q, \omega)= & \left(\frac{m}{q}\right)^{3} \frac{k_{F}^{6}}{(2 \pi)^{6}}\left[\mathcal{Q}_{\alpha \alpha^{\prime}}^{(2)}(0,0 ; \psi)-\mathcal{Q}_{\alpha \alpha^{\prime}}^{(2)}(0, \bar{q} ; \psi)-\mathcal{Q}_{\alpha \alpha^{\prime}}^{(2)}(\bar{q}, 0 ; \psi)+\mathcal{Q}_{\alpha \alpha^{\prime}}^{(2)}(\bar{q}, \bar{q} ; \psi)\right. \\
& \left.-\mathcal{Q}_{\alpha \alpha^{\prime}}^{(2)}(0,0 ; \psi+\bar{q})+\mathcal{Q}_{\alpha \alpha^{\prime}}^{(2)}(0,-\bar{q} ; \psi+\bar{q})+\mathcal{Q}_{\alpha \alpha^{\prime}}^{(2)}(-\bar{q}, 0 ; \psi+\bar{q})-\mathcal{Q}_{\alpha \alpha^{\prime}}^{(2)}(-\bar{q},-\bar{q} ; \psi+\bar{q})\right],
\end{aligned}
$$


where $^{1}$

$$
\begin{aligned}
\mathcal{Q}_{\alpha \alpha^{\prime}}^{(2)}\left(\bar{q}_{1}, \bar{q}_{2} ; \psi\right)= & \int_{-1}^{1} d y \frac{1}{2} \int_{0}^{1-y^{2}} d x \mathcal{G}_{\alpha}\left(x, y+\bar{q}_{1} ; \psi+\bar{q}_{1}\right) \\
& \times \frac{1}{\psi-y+i \eta_{\omega}} \mathcal{G}_{\alpha^{\prime}}\left(x, y+\bar{q}_{2} ; \psi+\bar{q}_{2}\right)
\end{aligned}
$$

and

$$
\mathcal{G}_{\alpha}(x, y ; \psi)=\int_{-1}^{1} d y^{\prime} \frac{1}{\psi-y^{\prime}+i \eta_{\omega}} W_{\alpha}^{\prime}\left(x, y ; y^{\prime}\right) .
$$

In the above expressions $\eta_{\omega} \equiv \operatorname{sign}(\omega) \eta, \bar{q} \equiv q / k_{F}$ is the transferred momentum in units of $k_{F}$, and $\psi$ is the Fermi gas scaling variable, which reads, for nonrelativistic kinematics,

$$
\psi=\frac{1}{k_{F}}\left(\frac{\omega m}{q}-\frac{q}{2}\right)
$$

The auxiliary functions $W^{\prime}$ and $W^{\prime \prime}$ are given in terms of the integral over the azimuthal angle of the momentum-dependent part of the interaction as

$$
\begin{gathered}
W_{\alpha}^{\prime}\left(x, y ; y^{\prime}\right)=\frac{1}{2} \int_{0}^{1-y^{\prime 2}} d x^{\prime} W_{\alpha}\left(x, y ; x^{\prime}, y^{\prime}\right), \quad(\mathrm{A} 7) \\
W_{\alpha}^{\prime \prime}\left(y, y^{\prime} ; \bar{q}\right)=\frac{1}{2} \int_{0}^{1-y^{2}} d x \frac{1}{2} \int_{0}^{1-y^{\prime 2}} d x^{\prime} W_{\alpha}\left(x, y+\bar{q} ; x^{\prime}, y^{\prime}\right),
\end{gathered}
$$

where

$$
\begin{gathered}
W_{\alpha}\left(x, y ; x^{\prime}, y^{\prime}\right)=\int_{0}^{2 \pi} \frac{d \varphi}{2 \pi} V_{\alpha}\left(\boldsymbol{k}-\boldsymbol{k}^{\prime}\right) \\
W_{\alpha}\left(x, y ; x^{\prime}, y^{\prime}\right)=\int_{0}^{2 \pi} \frac{d \varphi}{2 \pi} V_{\alpha}\left(\boldsymbol{k}-\boldsymbol{k}^{\prime}\right) S_{z z}\left(\widehat{\boldsymbol{k}-\boldsymbol{k}^{\prime}}\right)
\end{gathered}
$$

for the nontensor and tensor terms, respectively, and $S_{z z}(\hat{k})=$ $3 \hat{\boldsymbol{k}}_{z} \hat{\boldsymbol{k}}_{z}-1$. The $x$ and $y$ variables are defined in terms of the momentum $\mathbf{k}$ as $y=k \cos \theta$ and $x=k^{2}-y^{2}$.

Employing a Gaussian interaction, only Eq. (A9) can be calculated analytically (in terms of a modified Bessel function of the first kind), so that in general one has to cope with multidimensional numerical integrations in the presence of (integrable) singularities. We calculated these integrals using a mix of deterministic and Monte Carlo [67] techniques: reaching a good accuracy turns out to be quite time consuming. A faster way of performing these calculations is provided by fitting the Gogny potentials in terms of meson exchanges. It turns out that a good fit of all the potentials employed in this work, for momenta up to $1 \mathrm{GeV} / c$, can be obtained through the exchange of the $\pi, \sigma, \rho$, and $\omega$ mesons, with standard dipole form factors, using the four coupling constants

\footnotetext{
${ }^{1}$ This expression is valid for central-central and central-tensor terms. About tensor-tensor contributions see below.
}

as fitting parameters. By using meson-exchange potentials, Eqs. (A7)-(A10) can be calculated analytically (see Ref. [29] for details), with a substantial improvement in computing time. As a cross-check the calculations shown in the paper were performed using both techniques.

Here, for completeness, we provide also the explicit expressions for the second-order tensor-tensor contributions in the CF expansion, which were not shown in Ref. [29]. When both the interaction lines in Fig. 1(c) contain a tensor term, the resulting contributions cannot be expressed in a factorized form as in Eq. (A4), but rather as a sum of factorized terms:

$$
\begin{aligned}
\mathcal{Q}_{\alpha \alpha^{\prime}}^{(2)} & \left(\bar{q}_{1}, \bar{q}_{2} ; \psi\right) \\
= & 2 \int_{-1}^{1} d y \frac{1}{2} \int_{0}^{1-y^{2}} d x \frac{1}{\psi-y+i \eta_{\omega}} \\
& \times\left[\mathcal{G}_{\alpha}^{(a)}\left(x, y+\bar{q}_{1} ; \psi+\bar{q}_{1}\right) \mathcal{G}_{\alpha^{\prime}}^{(a)}\left(x, y+\bar{q}_{2} ; \psi+\bar{q}_{2}\right)\right. \\
& +\mathcal{G}_{\alpha}^{(b)}\left(x, y+\bar{q}_{1} ; \psi+\bar{q}_{1}\right) \mathcal{G}_{\alpha^{\prime}}^{(c)}\left(x, y+\bar{q}_{2} ; \psi+\bar{q}_{2}\right) \\
& +\mathcal{G}_{\alpha}^{(c)}\left(x, y+\bar{q}_{1} ; \psi+\bar{q}_{1}\right) \mathcal{G}_{\alpha^{\prime}}^{(b)}\left(x, y+\bar{q}_{2} ; \psi+\bar{q}_{2}\right) \\
& \left.-\mathcal{G}_{\alpha}^{(d)}\left(x, y+\bar{q}_{1} ; \psi+\bar{q}_{1}\right) \mathcal{G}_{\alpha^{\prime}}^{(d)}\left(x, y+\bar{q}_{2} ; \psi+\bar{q}_{2}\right)\right], \\
\alpha, \alpha^{\prime} & =t, t \tau,
\end{aligned}
$$

with

$$
\begin{array}{r}
\mathcal{G}_{\alpha}^{(l)}(x, y ; \psi)=\int_{-1}^{1} d y^{\prime} \frac{1}{\psi-y^{\prime}+i \eta_{\omega}} W_{\alpha}^{(l)}\left(x, y ; y^{\prime}\right), \\
W_{\alpha}^{(l)}\left(x, y ; y^{\prime}\right)=\frac{1}{2} \int_{0}^{1-y^{\prime 2}} d x^{\prime} W_{\alpha}^{(l)}\left(x, y ; x^{\prime}, y^{\prime}\right),
\end{array}
$$

and

$$
\begin{array}{r}
W_{\alpha}^{(a)}\left(x, y ; x^{\prime}, y^{\prime}\right)=\int_{0}^{2 \pi} \frac{d \varphi}{2 \pi} V_{\alpha}\left(\boldsymbol{k}-\boldsymbol{k}^{\prime}\right) \frac{h()-2\left(y^{\prime}-y\right)^{2}}{h()+\left(y^{\prime}-y\right)^{2}}, \\
W_{\alpha}^{(b)}\left(x, y ; x^{\prime}, y^{\prime}\right)=\int_{0}^{2 \pi} \frac{d \varphi}{2 \pi} V_{\alpha}\left(\boldsymbol{k}-\boldsymbol{k}^{\prime}\right), \\
W_{\alpha}^{(c)}\left(x, y ; x^{\prime}, y^{\prime}\right)=\int_{0}^{2 \pi} \frac{d \varphi}{2 \pi} V_{\alpha}\left(\boldsymbol{k}-\boldsymbol{k}^{\prime}\right) \frac{2 h()-\left(y^{\prime}-y\right)^{2}}{h()+\left(y^{\prime}-y\right)^{2}}, \\
W_{\alpha}^{(d)}\left(x, y ; x^{\prime}, y^{\prime}\right)=\int_{0}^{2 \pi} \frac{d \varphi}{2 \pi} V_{\alpha}\left(\boldsymbol{k}-\boldsymbol{k}^{\prime}\right) \frac{h()\left(y^{\prime}-y\right)}{h()+\left(y^{\prime}-y\right)^{2}},
\end{array}
$$

having defined

$$
h()=h\left(x, x^{\prime}, \varphi\right)=x^{\prime}+x-2 \sqrt{x^{\prime}} \sqrt{x} \cos (\varphi) .
$$

Again, for Gaussian potentials all the integrations have to be performed numerically, whereas for meson-exchange potentials the expressions (A13)-(A17) can be obtained analytically. 
[1] W. M. Alberico, M. Ericson, and A. Molinari, Nucl. Phys. A 379, 429 (1982).

[2] S. Krewald, K. Nakayama, and J. Speth, Phys. Rep. 161, 103 (1988).

[3] A. De Pace and M. Viviani, Phys. Rev. C 48, 2931 (1993).

[4] A. Gil, J. Nieves, and E. Oset, Nucl. Phys. A 627, 543 (1997).

[5] A. De Pace, C. Garcia-Recio, and E. Oset, Phys. Rev. C 55, 1394 (1997).

[6] M. Ichimura, H. Sakai, and T. Wasaka, Prog. Part. Nucl. Phys. 56, 446 (2006).

[7] M. Martini, M. Ericson, G. Chanfray, and J. Marteau, Phys. Rev. C 80, 065501 (2009).

[8] C. Garcia-Recio, J. Navarro, N. Van Giai, and L. L. Salcedo, Ann. Phys. 214, 293 (1992).

[9] J. Margueron, J. Navarro, and N. Van Giai, Phys. Rev. C 74, 015805 (2006).

[10] D. Davesne, M. Martini, K. Bennaceur, and J. Meyer, Phys. Rev. C 80, 024314 (2009); 84, 059904 (2011).

[11] A. Pastore, D. Davesne, Y. Lallouet, M. Martini, K. Bennaceur, and J. Meyer, Phys. Rev. C 85, 054317 (2012).

[12] A. Pastore, M. Martini, V. Buridon, D. Davesne, K. Bennaceur, and J. Meyer, Phys. Rev. C 86, 044308 (2012).

[13] D. Davesne, A. Pastore, and J. Navarro, Phys. Rev. C 89, 044302 (2014).

[14] A. Pastore, M. Martini, D. Davesne, J. Navarro, S. Goriely, and N. Chamel, Phys. Rev. C 90, 025804 (2014).

[15] D. Davesne, J. W. Holt, A. Pastore, and J. Navarro, Phys. Rev. C 91, 014323 (2015).

[16] A. Pastore, D. Davesne, and J. Navarro, Phys. Rep. 563, 1 (2014).

[17] T. Lesinski, K. Bennaceur, T. Duguet, and J. Meyer, Phys. Rev. C 74, 044315 (2006).

[18] V. Hellemans, A. Pastore, T. Duguet, K. Bennaceur, D. Davesne, J. Meyer, M. Bender, and P.-H. Heenen, Phys. Rev. C 88, 064323 (2013).

[19] A. Pastore, D. Tarpanov, D. Davesne, and J. Navarro, Phys. Rev. C 92, 024305 (2015).

[20] N. Iwamoto and C. J. Pethick, Phys. Rev. D 25, 313 (1982).

[21] S. Reddy, M. Prakash, J. M. Lattimer, and J. A. Pons, Phys. Rev. C 59, 2888 (1999).

[22] J. Navarro, E. S. Hernandez, and D. Vautherin, Phys. Rev. C 60, 045801 (1999).

[23] D. Gogny and R. Padjen, Nucl. Phys. A 293, 365 (1977).

[24] J. Margueron, N. V. Giai, and J. Navarro, Phys. Rev. C 72, 034311 (2005).

[25] W. M. Alberico, M. B. Barbaro, A. De Pace, T. W. Donnelly, and A. Molinari, Nucl. Phys. A 563, 605 (1993).

[26] M. B. Barbaro, A. De Pace, T. W. Donnelly, and A. Molinari, Nucl. Phys. A 569, 701 (1994).

[27] M. B. Barbaro, A. De Pace, T. W. Donnelly, and A. Molinari, Nucl. Phys. A 596, 553 (1996).

[28] M. B. Barbaro, A. De Pace, T. W. Donnelly, and A. Molinari, Nucl. Phys. A 598, 503 (1996).

[29] A. De Pace, Nucl. Phys. A 635, 163 (1998).

[30] J. Margueron, J. Navarro, N. Van Giai, and P. Schuck, Phys. Rev. C 77, 064306 (2008).

[31] D. Gogny, in Proceedings of the International Conference on Nuclear Physics, Munich, 1973, edited by J. de Boer and H. J. Mang (North-Holland, Amsterdam, 1974), p. 48.

[32] J. F. Berger, M. Girod, and D. Gogny, Comput. Phys. Commun. 63, 365 (1991).

[33] F. Chappert, M. Girod, and S. Hilaire, Phys. Lett. B 668, 420 (2008).
[34] S. Goriely, S. Hilaire, M. Girod, and S. Peru, Phys. Rev. Lett. 102, 242501 (2009).

[35] T. Otsuka, T. Matsuo, and D. Abe, Phys. Rev. Lett. 97, 162501 (2006).

[36] M. Anguiano, G. Co', V. De Donno, and A. M. Lallena, Phys. Rev. C 83, 064306 (2011).

[37] M. Anguiano, M. Grasso, G. Co', V. De Donno, and A. M. Lallena, Phys. Rev. C 86, 054302 (2012).

[38] V. De Donno, G. Co', M. Anguiano, and A. M. Lallena, Phys. Rev. C 90, 024326 (2014).

[39] A. Pastore, D. Davesne, and J. Navarro, J. Phys. G 41, 055103 (2014).

[40] J. Navarro and A. Polls, Phys. Rev. C 87, 044329 (2013).

[41] A. L. Fetter and J. D. Walecka, Quantum Theory of ManyParticle Systems (McGraw-Hill, New York, 1971).

[42] A. A. Abrikosov, L. P. Gorkov, and I. E. Dzyaloshinski, Methods of Quantum Field Theory in Statistical Physics (Dover, New York, 1963).

[43] F. Lenz, E. J. Moniz, and K. Yazaki, Ann. Phys. (NY) 129, 84 (1980).

[44] H. Feshbach, Theoretical Nuclear Physics: Nuclear Reactions (Wiley, New York, 1992).

[45] A. Dellafiore, F. Lenz, and F. A. Brieva, Phys. Rev. C 31, 1088 (1985).

[46] F. A. Brieva and A. Dellafiore, Phys. Rev. C 36, 899 (1987).

[47] P. Schuck, R. W. Hasse, J. Jaenicke, C. Grégoire, B. Rémaud, F. Sébille, and E. Suraud, Prog. Part. Nucl. Phys. 22, 181 (1989).

[48] J. Ventura, A. Polls, X. Vias, and E. S. Hernandez, Nucl. Phys. A 578, 147 (1994).

[49] D. Lopez-Val, A. Rios, A. Polls, and I. Vidana, Phys. Rev. C 74, 068801 (2006).

[50] R. Sellahewa and A. Rios, Phys. Rev. C 90, 054327 (2014).

[51] S. Peru and M. Martini, Eur. Phys. J. A 50, 88 (2014).

[52] M. Martini, S. Peru, S. Hilaire, S. Goriely, and F. Lechaftois, Phys. Rev. C 94, 014304 (2016).

[53] M. Martini, S. Peru, and S. Goriely, Phys. Rev. C 89, 044306 (2014).

[54] K. Nakayama, S. Krewald, J. Speth, and W. G. Love, Nucl. Phys. A 431, 419 (1984).

[55] T. Shigehara, K. Shimizu, and A. Arima, Nucl. Phys. A 492 , 388 (1989).

[56] T. Shigehara, K. Shimizu, and A. Arima, Nucl. Phys. A 510, 106 (1990).

[57] M. Martini, M. Ericson, G. Chanfray, and J. Marteau, Phys. Rev. C 81, 045502 (2010).

[58] A. B. Migdal, Theory of Finite Fermi Systems and Applications to Atomic Nuclei (Interscience, New York, 1967).

[59] E. Bauer and G. Co', J. Phys. G 27, 1813 (2001).

[60] M. Martini, N. Jachowicz, M. Ericson, V. Pandey, T. Van Cuyck, and N. Van Dessel, Phys. Rev. C 94, 015501 (2016).

[61] V. De Donno, G. Co', M. Anguiano, and A. M. Lallena, Phys. Rev. C 83, 044324 (2011).

[62] V. De Donno, G. Co', M. Anguiano, and A. M. Lallena, Phys. Rev. C 93, 034320 (2016).

[63] F. Chappert, N. Pillet, M. Girod, and J.-F. Berger, Phys. Rev. C 91, 034312 (2015).

[64] G. Bertsch, J. Borysowicz, H. McManus, and W. G. Love, Nucl. Phys. A 284, 399 (1977).

[65] H. Nakada, Phys. Rev. C 68, 014316 (2003).

[66] J. Dobaczewski, K. Bennaceur, F. Raimondi, K. Bennaceur, and F. Raimondi, J. Phys. G 39, 125103 (2012).

[67] T. Hahn, Comput. Phys. Commun. 168, 78 (2005). 